\title{
BMJ Open Prevalence of common mental health disorders in adults who are high or costly users of healthcare services: protocol for a systematic review and meta-analysis
}

Ferozkhan Jadhakhan, ${ }^{1,2}$ Oana C Lindner, ${ }^{3}$ Amy Blakemore, ${ }^{\odot 4}$ Elspeth Guthrie $^{\oplus 3}$

To cite: Jadhakhan $F$, Lindner OC, Blakemore A, et al. Prevalence of common mental health disorders in adults who are high or costly users of healthcare services: protocol for a systematic review and meta-analysis. BMJ Open 2019;9:e028295. doi:10.1136/ bmjopen-2018-028295

- Prepublication history and additional material for this paper are available online. To view these files, please visit the journal online (http://dx.doi. org/10.1136/bmjopen-2018028295).

FJ and OCL are joint first authors.

Received 30 November 2018 Revised 15 July 2019 Accepted 24 July 2019

Check for updates

(C) Author(s) (or their employer(s)) 2019. Re-use permitted under CC BY-NC. No commercial re-use. See rights and permissions. Published by BMJ.

For numbered affiliations see end of article.

Correspondence to Dr Ferozkhan Jadhakhan; F.Jadhakhan@bham.ac.uk

\section{ABSTRACT}

Introduction In all healthcare settings, a small proportion of patients account for a large level of healthcare use and associated high healthcare costs. Depression and anxiety are common co-morbidities in patients who are high users of care. The aims of this systematic review are to: (1) estimate the prevalence of anxiety/depression in adults who are high users of general physical healthcare services and/or who accrue high healthcare costs (2) estimate the magnitude of healthcare use associated with the presence of anxiety/depression.

Methods and analysis This review will include any studies where patients are high users of primary, secondary or emergency healthcare services and/or accrue high healthcare costs. This is the first systematic review to focus on patients who are over the age of 18 , whose degree of anxiety/depression has been evaluated with a standardised questionnaire or by a clinical interview generating a diagnosis according to international diagnostic criteria. The review will include eligible studies indexed in Medline, PsychINF0, Embase, Cumulative Index to Nursing and Allied Health Literature, Prospective Register of Systematic Reviews, Cochrane Library from inception to 1 April 2019. We will estimate the prevalence of anxiety/depression in these populations and the magnitude of use associated with anxiety/depression across various general physical healthcare settings. We will provide a narrative description of findings and factors that may influence them. A meta-analysis may be pursued if the degree of heterogeneity across studies is acceptable. Ethics and dissemination This systematic review will use data from existing studies, hence no ethical approvals are required. Findings will be disseminated in a peerreviewed publication and at relevant academic meetings. PROSPERO registration number PROSPERO CRD42018102628.

\section{INTRODUCTION}

The cost of healthcare in developed countries has continued to grow over the recent years, and the current projected trajectories of growth are unsustainable. ${ }^{1}$ This situation is particularly severe in the USA, where the cost of healthcare is nearly twice that of most
Strengths and limitations of this study

- This systematic review will include both studies of high healthcare use and/or high healthcare costs.

- It will include studies undertaken in general physical healthcare settings-primary, secondary care and emergency departments.

- It will focus on studies that have specifically recorded the presence of depression and/or anxiety in the high cost/high use population studied, using standardised questionnaires or clinical interviews leading to a clinical diagnosis.

- We will provide a narrative summary of findings with sources of variation and bias based on a comprehensive data extraction framework, with relevant subgroup analyses and interpretations based on: country, type of healthcare system, location of study (primary, secondary care, emergency department or total healthcare) and way of recording depression/ anxiety.

- A meta-analysis may not be feasible given a likely high level of heterogeneity in outcome definitions and measurements.

other developed countries. ${ }^{12}$ Across healthcare systems, a small proportion of patients account for a large proportion of healthcare use and cost. ${ }^{3}$ These findings have consistently emerged from studies of general practice (GP) attendances, ${ }^{4}$ inpatient length of stay, ${ }^{5} 6$ outpatient appointments ${ }^{7}$ and emergency department (ED) services. ${ }^{8-10}$ In primary care, approximately $10 \%$ of 'frequent attenders' account for up to $39 \%$ of all consultations. ${ }^{11}$ In the USA, approximately $5 \%$ of patients account for about $50 \%$ of all US healthcare spending. ${ }^{12}$

It has been suggested that approximately $50 \%$ of high users of healthcare in primary and secondary care have significant mental health problems, either alone or, in addition to physical health needs, and have been 
termed 'distressed high users'. ${ }^{13}$ High use of healthcare services has been associated with a variety of mental health problems including multiple psychiatric diagnoses, ${ }^{14} 15$ long histories of psychological ill health, ${ }^{16}{ }^{17}$ history of childhood abuse or neglect ${ }^{18}$ or addictions. ${ }^{19}$

A recent systematic review of the general characteristics of high-cost patients found a high prevalence of multiple chronic conditions among this patient population. ${ }^{20}$ Mental health problems were also common but varied according to the healthcare system. In US Medicaid, the prevalence of mental illness ranged from $30 \%$ to $75 \%$, whereas in US Medicare, the prevalence was between $10 \%$ and $25 \%$. One of the main findings of the review was that high-cost patients were more likely to have a mental health disorder. There were, however, no details as to the nature of mental health problems experienced by these high-cost patients, as data were grouped under a broad category of mental and behavioural disorders. This review will focus on patients with depression and anxiety disorders, as they are the most common form of mental disorder. We will focus on studies where depression and anxiety are identified through standardised questionnaires or by clinical interviews leading to a clinical diagnosis. Our review will provide information about the prevalence of depression/anxiety in both high-income and low-income countries and in different general physical healthcare settings, namely primary, secondary care and ED.

Several methods have been studied to try to improve the care of high-cost or high-use patients in the hope of reducing excessive or unnecessary healthcare use, but efforts to date have had mixed results. ${ }^{21}{ }^{22}$ Evidence suggests that effectiveness and efficiency of care improves when interventions are targeted to those who are most likely to benefit. ${ }^{23}{ }^{24}$ Specific interventions for treating depression and anxiety in people with comorbid physical health problems have shown promising results ${ }^{25}{ }^{26}$ but have not been targeted at high-cost patients with comorbid depression/anxiety.

Improved recognition of the association of depression and anxiety with high healthcare use and costs will enable treatments that have already been developed for depression/anxiety in physical disease to be evaluated in this high need/high cost group. Although there has been a general call for better integration of physical and mental health services, the treatment and management of comorbid depression/anxiety in chronic physical disease remains poorly managed. ${ }^{27}$

Our aim is to estimate the prevalence of anxiety/depression in adults who are high users of healthcare or accrue high healthcare costs and where possible to estimate the magnitude of use associated with anxiety/depression. Segmentation analysis has been suggested as a method to identify homogeneous groups of patients with similar characteristics, needs and behaviours to personalise treatment and policy. ${ }^{28}$ We are specifically interested in depression and anxiety, as opposed to all mental health problems, as interventions have already been developed to treat depression/anxiety when associated with physical disease. Such interventions could be used to target a subgroup of high-use/high-cost patients with the potential to improve their health and reduce healthcare use. Other forms of mental illness require other treatment approaches.

\section{Aims}

This systematic review will aim to: (1) estimate the prevalence of anxiety and/or depression in patients who are high users of healthcare or accrue high healthcare costs and (2) determine the magnitude of healthcare use/cost associated with the presence of anxiety and/or depression.

\section{METHODS AND DESIGN Population}

This review will include studies focusing on adults aged $\geq 18$ years, who are high users of healthcare services or accrue high healthcare costs and whose level of depression/anxiety have been evaluated through standardised questionnaires or clinical interviews. We include studies conducted in general rather than specialist physical health services, namely primary, secondary care and ED across all healthcare systems. We will not include studies with populations seen in the context of psychiatric or mental health services for a primary diagnosis of a psychiatric condition (ie, psychosis, schizophrenia) as the aim is to estimate the prevalence of anxiety/depression among high users of general physical healthcare services. We will not include specific medical specialties/illnesses associated with more frequent or costly healthcare use due to the nature of the condition or specialty (eg, surgery, paediatrics, palliative care, obstetrics, transplant, neurodegenerative diseases, oral and maxillofacial, dentistry, nephrology, infectious diseases, virology and HIV/AIDS studies, physiotherapy and cosmetic surgery).

We have focused on general hospital, ED and primary care services to ensure the review is relevant to as wide a population as possible. There is great variability in the way costs, healthcare use and depression/anxiety have been recorded in the literature. To add studies on individual disease conditions or specialities would considerably inflate the variability within the population of this review.

For studies of high-cost patients, we will include studies that have defined high-cost patients as being in the top 1 st, 5th, 10th and 20th percentiles of the patient population. ${ }^{20}$ For studies involving high use of healthcare, we will include studies that have either used similar percentiles to describe high use (ie, 1st, 5th, 10th or 20th) or have used a recognised definition of high or frequent use for the particular healthcare services. For ED, we will use the definition of four or more attendances per annum. ${ }^{29}$ For primary care, we will use the definition of 10 or more attendances per year ${ }^{30}$ or the top $10 \%$ of consulters. ${ }^{31}$ 
The review will include studies reporting costs and healthcare use. However, resource use and costs are sensitive to variability both within and between countries due to aspects such as local prices or aspects of service organisation and delivery. This may limit the generalisability and transferability of estimates of cost and healthcare across settings. We will not attempt to combine costs or health use in the analyses across studies. The prevalence of depression or anxiety will be compared across studies. To determine the magnitude of healthcare use associated with depression/anxiety in high-use/high-cost patients, we will estimate the healthcare used by depressed and non-depressed individuals. If sufficient studies report similar effect measures (eg, odds ratio ORs, relative risk, incidence rate ratios) of the frequency of healthcare use in these patients, ${ }^{32}$ they will be combined in a meta-analysis, consistent with current recommendations. ${ }^{33-35}$ Studies reporting different effect measures will not be combined, unless they can be transformed. ${ }^{34}{ }^{35}$

\section{Interventions}

We will not include randomised controlled trials due to their selective nature. We will include cohort studies of naturalistic changes in health service delivery for example, implementation of a new integrated care pathway across a geographical region, where external validity is likely to be high.

\section{Comparators}

We will include studies where anxiety/depression is described in groups of patients considered 'high/ frequent users' and/or 'high cost users' versus non-high cost and non-high users of healthcare services. We will include studies where high healthcare use/costs are compared between patients with anxiety/depression versus study patients without anxiety/depression.

\section{Outcomes}

The primary outcome is the prevalence of anxiety/ depression in high/frequent and/or costly users of general healthcare services. The secondary outcome is the magnitude of healthcare use and costs associated with anxiety/depression. Studies including a diverse range of standardised assessments and metrics for anxiety/ depression will be eligible. We will extract and report the prevalence of anxiety/depression based on the type of assessments used. For standardised, validated, self-report measures, this will be in the form of caseness. For clinical interviews, this will be in the form of a clinical diagnosis. Studies will be excluded if they do not meet our criteria for the assessment of anxiety or depression. A review concerning general mental health disorders has already been undertaken by Wammes $e t a l .{ }^{20}$

\section{Study designs}

We will include retrospective and prospective cohort studies, case-control, nested case-control and cross-sectional studies. We will exclude case studies, randomised controlled trials and qualitative studies.

\section{Search strategy}

We will screen the five databases that are most likely to include studies focusing on our outcomes of interest: Medline, PsychINFO, Embase, Cumulative Index to Nursing and Allied Health Literature, Prospective Register of Systematic Reviews, Cochrane Library, from inception to 1 April 2019. We will hand-search reference lists of relevant reviews/meta-analyses. For each database our search strategy has three parts (see search terms for Medline in online supplementary appendix 1). Search terms within the first part will identify studies pertaining to general healthcare settings of interest. The second part will focus on terms related to high cost or high/frequent use of healthcare services. The final part will focus the search on studies evaluating anxiety/ depression. This strategy ensures we identify all studies (1) conducted across general healthcare settings such as primary, secondary care and ED; (2) which include measurements of healthcare use and/or costs (3) and assess anxiety/depression. We will not be able to include studies that do not quantify either healthcare use or costs and studies that do not quantify anxiety/depression. This strategy ensures we include cohort studies describing the characteristics of high-use and/or high-cost patients and case-control studies where (1) anxiety/depression is compared between high and low use and/or costs, as defined by the respective study or where (2) healthcare use/costs is compared between patients with high and low levels of anxiety/depression, as defined by the study.

The strategy was developed in collaboration with experts in these fields and experienced librarians at the universities of Birmingham and Manchester to ensure it yields appropriate studies. We will include studies in all languages; translations will be pursued either by coauthors or by international colleagues/students in the universities of Birmingham, Leeds and Manchester. The search will be restricted to studies with adults over the age of 18 .

\section{Eligibility screening}

Eligible studies identified in all the databases will be organised using the EndNote reference management software. Duplicates will be identified and removed before screening titles and abstracts.

\section{Study selection}

Titles and abstracts will be screened independently by two reviewers. Remaining full-text articles will be further screened and evaluated for their eligibility using the adapted Hayden $e t a l$ s framework ${ }^{36}$ (see online supplementary appendix 2). Any disagreement over eligibility will be resolved through discussions with a third reviewer. The inclusion criteria checklist (table 1 and online supplementary appendix 2) ensures consistency in the review process and adherence to the Preferred Reporting Items for Systematic Reviews and Meta-Analyses (PRISMA) guidelines ${ }^{37}$; we will provide a PRISMA 


\section{Table 1 Inclusion criteria checklist based on the PRISMA guidelines}

\begin{tabular}{|c|c|}
\hline $\begin{array}{l}\text { Study } \\
\text { designs }\end{array}$ & $\begin{array}{l}\text { Cohort studies (retrospective and } \\
\text { prospective). } \\
\text { Case-control and nested case-control } \\
\text { studies. } \\
\text { Cross-sectional studies. }\end{array}$ \\
\hline Participants & $\begin{array}{l}\text { Adult aged } \geq 18 \text { years. } \\
\text { High user of healthcare. } \\
\text { Accrue high healthcare costs. } \\
\text { Assessment of anxiety/depression. }\end{array}$ \\
\hline Comparators & $\begin{array}{l}\text { Non-high cost and non-high users of } \\
\text { healthcare. } \\
\text { Frequent/high-cost users without depression/ } \\
\text { anxiety. }\end{array}$ \\
\hline Outcomes & $\begin{array}{l}\text { Prevalence of anxiety/depression in high } \\
\text { users of healthcare and/or high-cost patients. } \\
\text { Magnitude of cost or use of healthcare } \\
\text { associated with the presence of anxiety/ } \\
\text { depression. }\end{array}$ \\
\hline
\end{tabular}

PRISMA, Preferred Reporting Items for Systematic Reviews and Meta-Analyses.

flow-chart depicting the study selection and inclusion/ exclusion process.

\section{Quality assessment}

The quality of the included studies will first be ensured through the robustness of our database search and the careful title, abstract and full-text screening of relevant studies, carried out independently by two reviewers using the forms in online supplementary appendix 2 . We will only include studies reporting on high or costly users of healthcare where anxiety/depression is also assessed. All full-text studies meeting the eligibility criteria will undergo a quality assessment carried out independently by two reviewers through an adapted Newcastle-Ottawa Scale $^{38}$ (NOS; see online supplementary appendix 3). Assessment of study quality will include sampling method, sample size, adequacy of description of study population, attrition, method of outcome evaluation (eg, methods for recording costs/use; type of anxiety/ depression measurements, whether they are validated for the setting and so on), analytical method and consideration of confounders/covariates. The adapted NOS quality assessment form will first be piloted on known papers to ascertain its feasibility. Opinion differences will be resolved by consensus or by involving a third reviewer. Risk of bias (including reporting bias) will be evaluated commensurate with recent recommendations for the narrative interpretation of variation in observational studies $^{34}{ }^{35}$ and the recommendations of the Cochrane Collaboration. ${ }^{39}{ }^{40}$ Risk of bias will be reported in a categorical format, with 'yes' indicating high risk, 'no' low risk or 'unclear' for each predefined domain. We will describe the study quality and risk of bias for each study included in our review. For both low-quality and high-quality studies, we will provide a narrative description of definitions and measurements of costs and healthcare use and prevalence of anxiety/depression used across healthcare settings, regions and patient populations. If a meta-analysis can be pursued, we will run a sensitivity analysis to explore if outcomes change when removing low-quality studies. Through sensitivity analyses, we will also specifically explore the effects of excluding studies which have used non-validated measures of depression/anxiety in medically ill populations.

\section{Data extraction}

Following the selection of relevant full-text articles and quality assessment, two reviewers will independently extract relevant information in a data extraction form designed based on Hayden et $a l$ s framework ${ }^{36}$ (see online supplementary appendix 2); it will be developed iteratively and first piloted on five known papers, by two reviewers, before performing the data extraction for all studies.

The data extraction form focuses on the study design, population, comparator and outcome. It will include: year and country of study, type of healthcare system, criteria used to define high use or high costs, method used to record depression/anxiety (self-report measure validated or non-validated, clinical interview), prevalence of depression and anxiety, healthcare use, costs and associated ranges, the methods used to evaluate these, healthcare settings (eg, primary, secondary or ED or total healthcare use/cost, if reported as general metrics), healthcare use and cost estimates for depressed/anxious patients compared with non-depressed/anxious patients and patient characteristics (eg, comorbidities, whether anxiety/depression is managed). We will also record the presence and source of bias, including funding, given its potential association with reporting bias. ${ }^{39} 40$

\section{DATA ANALYSIS AND SYNTHESIS}

The primary outcome is the prevalence of anxiety and/ or depression in patients who are high and/or costly users of healthcare services. Prevalence rates with any dispersion metrics will be extracted or calculated from the data available. Where enough studies are available for quantitative summaries (minimum two studies ${ }^{41}$ ), we will offer weighted estimates of prevalence within relevant subgroups related to populations, comparators, study designs, measurement types and geographical regions. Pooled prevalence estimates with $95 \%$ CIs will be calculated using SPSS V.25 (IBM Corp); where possible and warranted, estimate transformations and quantitative summaries will be pursued using $\mathrm{R}^{33}$

The secondary outcome is the magnitude of healthcare use orcost associated with the presence of anxiety/ depression. We are not attempting to pool or calculate costs or health use across studies. We will only be able to determine the magnitude of healthcare use associated with depression/anxiety in relation to studies that have 
specifically calculated or estimated these. This will be studies where high healthcare use/costs are compared between patients with anxiety/depression versus patients without anxiety/depression. Outcome metrics (including ORs, relative risk, etc) and 95\% CIs will be extracted from studies presenting the number of healthcare contacts (eg, ED attendances or GP contacts or number of hospital admissions) by subjects with and without depression.

We expect both the prevalence of depression/anxiety to be available from studies evaluating high-use/highcost populations alone or in studies comparing high-use/ high-cost patients to general patient populations or populations with low use/cost. Data analysis will result in quantitative and narrative summaries, as appropriate, based on current recommendations for the pooling of observational studies. ${ }^{34}{ }^{35}$ Whereas there is some published guidance on the number of studies necessary to ensure the power of the effect size estimates when pooling interventional studies, ${ }^{32} 42$ there are no similar clear, agreed guidelines on the number of studies necessary for an appropriately powered meta-analysis of observational studies. We will offer a quantitative summary for any number of studies (2>) if combining their outcomes is clinically meaningful, if they report the same effect metrics or if transformations are possible. ${ }^{34} 3541$ We will comment on these pooled results in light of clinical practice and research significance and potential statistical issues that may decrease the generalisability of the effect estimates (eg, high level of heterogeneity, potential sources of bias). For both outcomes, subgroups will be explored quantitatively and narratively, as appropriate and depending on the type of effect estimates available, based on potential differences related to: (1) country, (2) type of healthcare system, (3) medical settings (eg, primary, secondary care, ED, inpatients, outpatients, etc) and (4) metrics used to evaluate health use/costs (eg, attendances, hospital admissions, etc). For instance, we expect to find studies that may only focus on frequent attendance at ED, primary care outpatient visits, number of bed days in secondary care or more generic attendance metrics across either of these healthcare settings. We will account for such differences in reporting, but we are not planning to compare outcomes across settings, just to record and estimate the magnitude of use/cost in each of these contexts.

We will use random-effects models to describe the prevalence of depression/anxiety high-use or high-cost populations. This is because it is implausible that the underlying study-specific prevalence of depression (ie, the prevalence that would be observed were a study of infinite size) is the same for each study. Prevalence is likely to vary from study to study according to factors, both measured and unmeasured, that differ between them. ${ }^{43}$

We will use the inverse variance method of DerSimonian and Laird to estimate between-study heterogeneity in underlying depression prevalence and the $\mathrm{I}^{2}$ measure with associated $95 \%$ CIs, which represents the proportion of total variance attributable to this heterogeneity. ${ }^{39} 44$ The $\mathrm{I}^{2}$ measure gives the percentage of variability in the effect estimate that is due to heterogeneity rather than to chance. A rough guide to the interpretation of the $\mathrm{I}^{2}$ measure suggests that $\mathrm{I}^{2}<40 \%$ indicates low to no problems with heterogeneity, $30 \%$ to $60 \%$ indicates moderate problems, $60 \%$ to $90 \%$ indicates significant problems, whereas an $\mathrm{I}^{2}$ of $75 \%$ or more suggests considerable problems. ${ }^{42}$ If $\mathrm{I}^{2}$ is less than $40 \%$, we will consider the estimated effect to have a low degree of heterogeneity, but this will also be interpreted in light of the magnitude, direction of the effect and its $95 \% \mathrm{CI}$, sources of bias and clinical significance. ${ }^{35} 394142$

Egger's statistics with 95\% CIs and associated funnel plot will depict potential publication or small sample bias related to our main outcome summaries and/or within subgroups. ${ }^{45}$ Egger's test is based on the Galbraith plot which is a plot of study difference over standarderror against 1 /standarderror. Egger suggests calculating the regression of study difference over standarderror on $1 /$ standarderror be undertaken to test the null hypothesis that the intercept is equal to zero. If Egger's test is significant $(p<0.05)$, it means that the funnel plot is asymmetric and that smaller studies with smaller precision show larger effects sizes, suggesting bias. Sensitivity analyses will be pursued at minimum on high-quality/ low-quality studies on the use of unvalidated standardised questionnaires and use of structured clinical interviews. If enough studies are available, other factors that could influence our observed findings will be explored (eg, sample size). Tabular and narrative descriptions will be offered for the studies which cannot be pooled into quantitative summaries due to differing metrics.

\section{Patient and public involvement statement}

Patients and the public were not invited to contribute to the writing or editing of this systematic review protocol. The research question was informed by the lack of prior systematic reviews or meta-analyses exploring the outcomes of interest: prevalence of anxiety/depression in highly/costly healthcare users and the magnitude of healthcare use associated with anxiety/depression across adult populations in any general medical settings.

\section{DISCUSSION}

The purpose of this systematic review is to estimate the prevalence of anxiety/depression in people who are frequent, high-cost users of general healthcare services, and then, if possible, to estimate the level of healthcare use associated with the presence of anxiety/depression. While evidence is available suggesting that a small percentage of the population accrues a high percentage of healthcare/ costs, it is unclear to date to what extent the costs and use may be due to the presence of common mental health problems (depression/anxiety). By examining the information available to date, we aim to describe the prevalence of anxiety/depression in people who are highly/ costly healthcare users and where possible the magnitude 
of use or costs associated with these two common mental health problems.

Our review will build on the recent systematic review by Wammes and colleagues ${ }^{20}$ that described the characteristics of high-cost patients and found that a high prevalence of high-cost patients had associated mental health disorders. This review will specifically focus on depression/anxiety and include both studies of cost and healthcare use. It will also provide information about the prevalence of depression/anxiety in different healthcare settings, including primary care and ED. There is a trade-off between diagnostic accuracy versus size of study. Our results will complement those of Wammes and colleagues ${ }^{20}$ and increase our understanding of the role of depression/anxiety in driving healthcare use and costs.

Strengths of this review are that it focuses on common mental health problems, includes both studies of healthcare cost and healthcare use and includes general healthcare settings, including primary, secondary care and ED. Additional strengths are the inclusion of studies published in any language and the independent study identification, selection and data extraction pursued by two independent reviewers.

\section{Implications of results}

The results of this systematic review will provide an estimate of the prevalence of common mental health disorders in high users of healthcare services, while also providing an estimate of the magnitude of use associated with depression/anxiety. It will enable treatments, such as the collaborative care model, that have already been developed for the treatment of depression/anxiety in the physically ill, to be evaluated in high-cost patients with comorbid depression/anxiety resulting in a more personalised approach to both treatment and policy.

\section{Ethics and dissemination}

As this systematic review will use data from existing studies no ethical approvals are warranted; the results will bepublished in a peer-reviewed publication and presented at relevant academic meetings.

\footnotetext{
Author affiliations

${ }^{1}$ Institute of Mental Health, School of Psychology, College of Life and Environmental Sciences, University of Birmingham, Birmingham, West Midlands, UK

${ }^{2}$ Research and Innovation, Birmingham and Solihull Mental Health NHS Foundation Trust, Birmingham, West Midlands, UK

${ }^{3}$ Division of Psychological and Social Medicine, Leeds Institute of Health Sciences,

School of Medicine, University of Leeds, Leeds, West Yorkshire, UK

${ }^{4}$ Division of Nursing, Social Work and Midwifery, School of Health Sciences,

Manchester Academic Health Science Centre, University of Manchester, Manchester, UK
}

Acknowledgements The authors would like to thank Rosalind McNally (Outreach Librarian in the Research and Innovation Department and Knowledge Service in the Greater Manchester Mental Health NHS Foundation Trust) and Anita Phul (Librarian at the Barberry, National Centre for Mental Health, Birmingham and Solihull Mental Health NHS Foundation Trust, Birmingham) for their critical review of the search strategy.

Contributors OCL and FJ developed the search strategy. OCL drafted the manuscript and registered the protocol. $E G$ and $A B$ were involved in the design of the review and provided continuous feedback on the manuscript. OCL will be first reviewer and FJ will be second reviewer. All authors read and approved the manuscript.

Funding The authors have not declared a specific grant for this research from any funding agency in the public, commercial or not-for-profit sectors.

Competing interests None declared.

Patient consent for publication Not required.

Provenance and peer review Not commissioned; externally peer reviewed.

Open access This is an open access article distributed in accordance with the Creative Commons Attribution Non Commercial (CC BY-NC 4.0) license, which permits others to distribute, remix, adapt, build upon this work non-commercially, and license their derivative works on different terms, provided the original work is properly cited, appropriate credit is given, any changes made indicated, and the use is non-commercial. See: http://creativecommons.org/licenses/by-nc/4.0/.

\section{REFERENCES}

1. Porter ME. What is value in health care? $N$ Engl $J$ Med 2010;363:2477-81.

2. Davis K. 2012 Annual Report President's Message-Health Care Reform: A Journey, 2012. Available: https://www. commonwealthfund.org/publications/other-publication/2012/dec/ 2012-annual-report-presidents-message-health-care-reform? redirect source=/Publications/Annual-Report-Essays/2012/Dec/ Health-Care-Reform-A-Journey.aspx [Accessed 24 Apr 2014]

3. Rosella LC, Fitzpatrick T, Wodchis WP, et al. High-Cost health care users in Ontario, Canada: demographic, socio-economic, and health status characteristics. BMC Health Serv Res 2014;14:532.

4. Hochman M, Asch SM. Disruptive models in primary care: caring for High-Needs, high-cost populations. J Gen Intern Med 2017;32:392-7

5. Zook CJ, Moore FD. High-Cost users of medical care. N Engl J Med 1980;302:996-1002.

6. Calver J, Brameld KJ, Preen DB, et al. High-Cost users of hospital beds in Western Australia: a population-based record linkage study. Med J Aust 2006;184:393-7.

7. Kimerling R, Ouimette PC, Cronkite RC, et al. Depression and outpatient medical utilization: a naturalistic 10-year follow-up. Ann Behav Med 1999;21:317-21.

8. Ruger JP, Richter CJ, Spitznagel EL, et al. Analysis of costs, length of stay, and utilization of emergency department services by frequent users: implications for health policy. Acad Emerg Med 2004;11:1311-7.

9. Hunt KA, Weber EJ, Showstack JA, et al. Characteristics of frequent users of emergency departments. Ann Emerg Med 2006;48:1-8.

10. LaCalle E, Rabin E. Frequent users of emergency departments: the myths, the data, and the policy implications. Ann Emerg Med 2010;56:42-8.

11. Smits FT, Brouwer HJ, Zwinderman AH, et al. Morbidity and doctor characteristics only partly explain the substantial healthcare expenditures of frequent attenders: a record linkage study between patient data and reimbursements data. BMC Fam Pract 2013;14:138.

12. Schoenman JA, Chockley N. Understanding U.S. health care spending. Washington (DC), 2011. Available: http://www.nihcm.org/ images/stories/NIHCM-CostBriefEmail.pdf

13. Katon W, Von Korff M, Lin E, et al. Distressed high utilizers of medical care. DSM-III-R diagnoses and treatment needs. Gen Hosp Psychiatry 1990;12:355-62.

14. Patel S, Kai J, Atha C, et al. Clinical characteristics of persistent frequent attenders in primary care: case-control study. Fam Pract 2015;32:cmv076.

15. Reid S, Crayford T, Patel A, et al. Frequent attenders in secondary care: a 3-year follow-up study of patients with medically unexplained symptoms. Psychol Med 2003;33:519-24.

16. Von Korff M, Ormel J, Katon W, et al. Disability and depression among high utilizers of health care. A longitudinal analysis. Arch Gen Psychiatry 1992;49:91-100.

17. Arnow BA, Hunkeler EM, Blasey CM, et al. Comorbid depression, chronic pain, and disability in primary care. Psychosom Med 2006;68:262-8

18. Arnow BA. Relationships between childhood maltreatment, adult health and psychiatric outcomes, and medical utilization. J Clin Psychiatry 2004;65 Suppl 12:10-5.

19. Hensel JM, Taylor VH, Fung K, et al. Unique characteristics of highcost users of medical care with comorbid mental illness or addiction in a population-based cohort. Psychosomatics 2018;59:135-43. 
20. Wammes JJG, van der Wees PJ, Tanke MAC, et al. Systematic review of high-cost patients' characteristics and healthcare utilisation. BMJ Open 2018;8:e023113.

21. Blumenthal D, Anderson G, Burke S. Tailoring Complex-Care management, coordination, and integration for High-Need, highcost patients, 2016. Vital Dir Heal Heal Care Ser. Available: https:// nam.edu/wp-content/uploads/2016/09/Tailoring-Complex-CareManagement-Coordination-and-Integration-for-High-Need-HighCost-Patients.pdf

22. Hong CS, Siegel AL, Ferris TG. Caring for high-need, high-cost patients: what makes for a successful care management program? Commonw Fund 2014;19:1-20.

23. Brown RS, Peikes D, Peterson G, et al. Six features of Medicare coordinated care demonstration programs that cut hospital admissions of high-risk patients. Health Aff 2012;31:1156-66.

24. Anderson GF, Ballreich J, Bleich S, et al. Attributes common to programs that successfully treat high-need, high-cost individuals. Am J Manag Care 2015;21:e597-600.

25. Camacho EM, Ntais D, Coventry P, et al. Long-Term costeffectiveness of collaborative care (vs usual care) for people with depression and comorbid diabetes or cardiovascular disease: a Markov model informed by the COINCIDE randomised controlled trial. BMJ Open 2016;6:e012514.

26. Coventry PA, Hudson JL, Kontopantelis E, et al. Characteristics of effective collaborative care for treatment of depression: a systematic review and meta-regression of 74 randomised controlled trials. PLOS One 2014;9:e108114.

27. Department of Health and Social Care. No health without mental health, 2011. Available: https://assets.publishing.service.gov.uk/ government/uploads/system/uploads/attachment_data/file/138253/ dh_124058.pdf

28. Vuik SI, Mayer EK, Darzi A. Patient segmentation analysis offers significant benefits for integrated care and support. Health Aff 2016;35:769-75

29. Locker TE, Baston S, Mason SM, et al. Defining frequent use of an urban emergency department. Emerg Med J 2007;24:398-401.

30. Vedsted P, Christensen MB. Frequent attenders in general practice care: a literature review with special reference to methodological considerations. Public Health 2005;119:118-37.
31. Luciano JV, Fernández A, Pinto-Meza A, et al. Frequent attendance in primary care: comparison and implications of different definitions. Br J Gen Pract 2010;60:e49-55.

32. Valentine JC, Pigott TD, Rothstein HR. How many studies do you need?: a primer on statistical power for meta-analysis. J Educ Behav Stat 2009;35:215-47.

33. Chang B-H, Hoaglin DC. Meta-Analysis of odds ratios: current good practices. Med Care 2017;55:328-35.

34. Mueller M, D'Addario M, Egger M, et al. Methods to systematically review and meta-analyse observational studies: a systematic scoping review of recommendations. BMC Med Res Methodol 2018;18:44.

35. Dekkers OM, Vandenbroucke JP, Cevallos M, et al. COSMOS-E: guidance on conducting systematic reviews and meta-analyses of observational studies of etiology. PLoS Med 2019;16:e1002742.

36. Hayden JA, Côté P, Bombardier C. Evaluation of the quality of prognosis studies in systematic reviews. Ann Intern Med 2006;144:427-37.

37. Moher D, Shamseer L, Clarke M, et al. Preferred reporting items for systematic review and meta-analysis protocols (PRISMA-P) 2015 statement. Syst Rev 2015;4:1.

38. Hartling L, Milne A, Hamm MP, et al. Testing the Newcastle Ottawa scale showed low reliability between individual reviewers. J Clin Epidemiol 2013;66:982-93.

39. Higgins JP, Green S. Cochrane Handbook for systematic reviews of interventions version 5.1.0, 2011. Cochrane Collab. Available: www. cochrane-handbook.org[Accessed 15 Aug 2019].

40. Bero LA. Why the Cochrane risk of bias tool should include funding source as a standard item. Cochrane Database Syst Rev 2013;12:ED000075.

41. Ioannidis JPA, Patsopoulos NA, Rothstein HR. Reasons or excuses for avoiding meta-analysis in forest plots. BMJ 2008;336:1413-5.

42. Higgins JPT, Thompson SG, Deeks JJ, et al. Measuring inconsistency in meta-analyses. BMJ 2003;327:557-60.

43. Stroup DF, Berlin JA, Morton SC, et al. Meta-Analysis of observational studies in epidemiology: a proposal for reporting. meta-analysis of observational studies in epidemiology (MOOSE) group. JAMA 2000;283:2008-12.

44. DerSimonian R, Laird N. Meta-Analysis in clinical trials. Control Clin Trials 1986;7:177-88

45. Egger M, Davey Smith G, Schneider M, et al. Bias in meta-analysis detected by a simple, graphical test. BMJ 1997;315:629-34. 